\title{
Intraoperative In-vivo Verification of Tissues by Optical Spectroscopy
}

\author{
Dmitry Rogatkin ${ }^{1,2}$, Irina Raznitcyna ${ }^{1}$, Polina Glazkova $^{1}$ \\ ${ }^{1}$ Moscow Regional Research and Clinical Institute "MONIKI" named after M.F.Vladimirsky \\ Shepkina str. 61/2, Moscow, RF \\ rogatkin@monikiweb.ru; raznitsyna@medphyslab.com; polinikul@mail.ru \\ ${ }^{2}$ LLC "Research and Development Center EOS-Medica" \\ Nauchnyi proezd, 8, Moscow, RF
}

\section{Extended Abstract}

Any surgical intervention, even a minimal one, poses a potential danger, which is not always predictable. At laparoscopic and robot-assisted surgery, which are intensively developed today, the surgeon is almost devoid of tactile sensations. In addition, a colour reproduction of the image may be slightly altered. It leads to interpretive errors [1]. Another problem exists in regenerative medicine. At local transplantations of stem cells, the cells will differentiate into the tissue, they are injected. Thus, the necessity to create a convenient tool that allows the surgeon to perform an intraoperative rapid identification and verification of anatomical structures and types of tissues without its damaging is apparent. Non-invasive and minimally invasive optical diagnostic techniques, in particular, in-vivo spectrophotometric methods, are known in medicine for a long time, about a quarter of a century, or more [2]. In recent years, one can see many attempts to use them as intraoperative diagnostic tools for navigation and tissue identification at surgery interventions $[1,3]$. There are many studies devoted to the analysis of diffuse backscattering spectra for various tissues to find specific quantitative criteria for their differentiation [3]. There are also many researches attempting to verify tissues using fluorescence spectra [4] and autofluorescence ones [5,6].

In our study, we used the combined diffuse reflectance spectroscopy and fluorescent spectroscopy technique, which exploits the differences in absorption, scattering and fluorescent properties of biological tissues. The study was carried out with the use of our new laser diagnostic system "Multicom", developed by LLC "Research and Development Center EOSMedica". Optical properties of various types of tissues of laboratory white rats were measured. To reduce a number of variables, the principal component analysis with rotation (varimax) was applied. For the fluorescence spectrum, $98.3 \%$ of the dispersion was explained by five principal components. For the spectrum of the intensity of the diffuse backscattering, six principal components allowed to explain $92.2 \%$ of the dispersion. The eleven principal components obtained were included in the discriminant analysis. Statistical processing of data was carried out by IBM SPSS Statistics v23 (IBM corp., USA). Eight out of eleven types of tissues were verified with the accuracy more than $80 \%$. Thus, we showed experimentally that the combined spectroscopy technique may be useful for intraoperative navigation and identification of types of tissues.

\section{References}

[1] J. T. Elliott, A. V. Dsouza, S. C. Davis, J. D. Olson, K. D. Paulsen, D. W. Roberts, and B. W. Pogue, "Review of fluorescence guided surgery visualization and overlay techniques," Biomed. Opt. Exp., vol. 6, no. 10, pp. 3765-3782, 2015.

[2] D. Rogatkin, V. Shumskiy, S. Tereshenko and P. Polyakov, "Laser-based noninvasive spectrophotometry - an overview of possible medical application," Photon. Lasers Med., vol. 2, no. 3, pp. 225-240, 2013.

[3] A. Engelhardt, R. Kanawade, C. Knipfer, M. Schmid, F. Stelzle, W. Adler, "Comparing classification methods for diffuse reflectance spectra to improve tissue specific laser surgery," BMC med. Res. methodology, vol. 14, no. 1, pp. 91-116, 2014.

[4] E. N. Petritskaya, D. A. Kulikov, D. A. Rogatkin, I. A. Guseva, P. A. Kulikova, "Use of fluorescence spectroscopy for diagnosis of hypoxia and inflammatory processes in tissue," J. Opt. Technol., vol. 82, no. 12, pp. 810-814, 2015.

[5] F. Stelzle, C. Knipfer, W. Adler, M. Rohde, N. Oetter, E. Nkenke, M. Schmidt, and K. Tangermann-Gerk, "Tissue Discrimination by Uncorrected Autofluorescence Spectra: A Proof-of-Principle Study for Tissue-Specific Laser Surgery," Sensors, no. 13, pp. 13717-13731, 2013.

[6] O. D. Smirnova, D. A. Rogatkin, K. S. Litvinova, "Collagen as in vivo quantitative fluorescent biomarkers of abnormal tissue changes", J. of Innovative Opt. Health Sci., vol. 5, no. 2, 1250010, 2012. 